\title{
Optimal production control policy in unreliable batch processing manufacturing systems with transportation delay
}

\author{
B. Bouslah ${ }^{\mathrm{a}}, \mathrm{A}$. Gharbi $^{\mathrm{b}}$, R. Pellerina and A. Hajji ${ }^{\mathrm{c}}$ \\ a Department of Mathematics and Industrial Engineering, École Polytechnique de Montréal, \\ Montreal, Canada; \\ butomated Production Engineering Department, École de technologie supérieure, University of \\ Québec, Québec, Canada; \\ ${ }^{\mathrm{c}}$ Department of Operations and Decision Systems, Laval University, Québec, Canada
}

\begin{abstract}
This paper considers the problem of production planning of unreliable batch processing manufacturing systems. The finished goods are produced in lots, and are then transported to a storage area in order to continuously meet a constant demand rate. The main objective of this work is to jointly determine the optimal lot sizing and optimal production control policy that minimize the total expected cost of inventory/backlog and transportation, over an infinite time horizon. The decision variables are the lot sizing and the production rate. The problem is formulated with a stochastic dynamic programming model and the impulse control theory is applied to establish the Hamilton-Jacobi-Bellman (HJB) equations. Based on a numerical resolution of the HJB equations, it is shown that the optimal control policy is governed by a base stock policy for production rate control and economic lot size for batch processing. A thorough analysis and practical issues are addressed with a simulation based approach. Thus, a combined discrete-continuous simulation model is developed to determine the optimal parameters of the proposed policy when the failure and repair times follow general distributions. The results are illustrated with numerical examples and confirmed through sensitivity analysis.
\end{abstract}

Keywords Unreliable manufacturing systems, economic production quantity, feedback production planning, stochastic dynamic programming, simulation.

\section{Introduction}

The problem of the feedback production control of unreliable manufacturing systems has attracted many researchers. Among these, Kimemia and Gershwin (1983) introduced the concept of hedging point policy (HPP). Within such policy, an optimal inventory level named the hedging 
point is maintained during times of system availability in order to hedge against future system capacity shortages caused by failures. Akella and Kumar (1986) studied the problem of a singleproduct manufacturing system subject to stochastic breakdowns. They proved analytically the optimality of the HPP, and obtained an explicit solution for the hedging point minimizing the total expected discounted cost. The literature contains several research works that have addressed the properties and extensions of the HPP. We refer the reader to Sharifinia (1988), Gershwin (1994, 2000). All these works were based on the assumption of exponential failure and repair time distributions, and constant demand rate. However, Gharbi and Kenné (2000) extended the HPP to non-exponential failure and repair time distributions and/or random demand rate models using a simulation-based experimental approach. The use of simulation has been justified by the difficulties of applying the classical control theory for the non-Markovian models.

For many manufacturing systems, some significant delays (e.g., for handling, inspection, drying processes, transportation, etc.) may occur in the material flow. In the above mentioned models, these delays have been neglected when formulating and modeling the production control problem although they can affect the system performances. The notion of delays has however been considered on two others papers. First, Van Ryzin et al. (1991) examined the effects of delays in manufacturing systems that are modelled as continuous flow processes. They derived a heuristic control policy for a job shop problem with delays using theoretical arguments and approximations. Also, Mourani et al. (2008) studied a single-stage failure-prone manufacturing system where the produced material flow is added to a finished goods inventory after transportation. Considering that the production is controlled by a HPP, they focused on optimizing the continuous-flow model integrating the transportation delay.

The above cited research works deal with the item-by-item production problem and, do not address batch processing systems. Manufacturing systems facing a high demand rate, and in which the items produced need to be transported to a remote storage area before serving the demand, are commonly controlled by a batch production planning policy. These systems typically produce small-sized items (e.g., food and pharmaceutical products, small mechanical parts, etc.). In such cases, we should jointly determine the optimal production control policy and the economic manufacturing quantity (EMQ).

Many approaches have been developed to study the problem of EMQ and production control of unreliable manufacturing systems. Groenevelt et al. (1992a) focused on the impact of system 
breakdowns on production lot sizing decisions. Under the assumptions of deterministic constant production rate, negligible repair time, exponential failures and no backlogs, the authors determined the optimal lot sizing for two production reorder policies (no-resumption (NR) policy and abort/resume (AR) policy). In a subsequent paper, Groenevelt et al. (1992b) defined a production control policy to simultaneously determine the optimal lot sizing and the safety stock level that satisfy a prescribed service level. They assumed that, during a production run, a certain fraction of the items produced is instantaneously diverted into the safety stock. The proposed production policy can only be applied in the case of exponential failure time distribution. Kim and Hong (1997) and Kim et al. (1997) extended the Groenevelt et al. (1992a) model assuming that the times between failures are general distributions. Chung (1997) determined an approximate formula for the optimal lot sizing of the Groenevelt et al. (1992a) model by calculating its bounds. Abboud (1997) presented an approximate model to the EMQ problem considering that the number of failures during a production run is Poisson distributed and that the repair time follows a general distribution. Giri and Yun (2005) studied the EMQ problem for unreliable manufacturing systems subject to exponential failures and exponential/constant repair times, assuming that at most two failures can occur in a production cycle and that shortages are partially backlogged.

All the aforementioned works assume that the production rate is predetermined and constant. However, Giri et al. (2005) addressed the problem of EMQ for an unreliable production facility where the production rate is treated as a decision variable. They developed two models; with and without safety stock, in order to jointly determine the optimal lot sizing and the optimal feasible production rate. For the second model, the safety stock level is considered as a third decision variable. Giri and Dohi (2005) extended the Giri et al. (2005) model with safety stock, taking into account the preventive maintenance and assuming that the failure and repair times are general distributions. Sana and Chadhuri (2010) extended the Giri and Dohi (2005) model, considering the effect of an imperfect production process subject to random breakdowns. The proposed policy consists in determining the optimal safety stock, the optimal production rate and the optimal lot sizing. Many others studies have been interested in integrating preventive maintenance and/or quality issues in EMQ models. We refer readers to Hariga and Ben-Daya (1998), Salameh and Jaber (2000), Ben-Daya (2002), Chakraborty et al. (2009), and Liao et al. (2009). 
A critical assumption made throughout the EMQ literature is that the lot which is currently being processed can instantly meet the demand, and even build a safety stock if the difference between the production and demand rates is strictly positive. In reality, this assumption cannot be applied for a wide range of manufacturing systems where a certain transportation delay exists between the production facility and the final stock that truly serves the demand. The transportation may constitute material handling if the final storage area is a local warehouse, or a delivery for an external warehouse in a distribution chain. In both cases, the delay generally influences the production-inventory control policy, and should be considered when optimizing the entire system. As such, Li et al. (2009) developed an analytical approach based on the queuing theory for performance evaluation of a production-distribution system composed of a warehouse supplied by a manufacturing plant. The transportation delay is considered fixed, and the warehouse inventory is managed following a base-stock control policy. Their approach allows the determination of the optimal base-stock level without considering the production rate and the lot sizing as decision variables. Moreover, the transportation cost is neglected. It should be noted here that, in most EMQ models, the transportation cost of lots produced is not considered explicitly when calculating the total incurred cost. Sometimes, it is considered as a part of a fixed setup cost which does not depend directly on the lot sizing. However, Ertogral et al. (2007) considered the fact that transportation cost is a major part of the total operational cost, and they showed that production and inventory decisions are affected when transportation cost is considered explicitly in the EMQ model.

Recognizing the limitations of past EMQ models in considering the delay and cost of transportation, the main objective of this paper is to develop an integrated EMQ model and a feedback control policy of unreliable manufacturing systems in transportation context. Because finished goods are transported to the final stock in lots with separated delays, the problem cannot be represented with classical continuous-flow models. A stochastic dynamic programming based on the impulse control theory (Yong 1989, Yang 1999) is formulated in order to model the combined impulsive-continuous aspect of the problem. Since the analytical solution of the associated Hamilton-Jacobi-Bellman (HJB) equations is not generally available, a numerical resolution approach is adopted to determine the structure of the optimal control policy. The numerical resolution of the HJB equations in the case of negligible transportation delay shows that the production control policy leads to a combination of a modified HPP and a lot sizing 
control policy, which is extended to address the transportation delay problem. Given the limitations and the implementation difficulties inherent in the numerical resolution, we use an alternative control approach based on simulation. A combined discrete-continuous simulation model is developed to experimentally determine the optimal lot sizing and the optimal hedging level when the failure and repair times follow general distributions.

The remainder of the paper is organized as follows. In section 2, we define the notation used throughout the paper. Section 3 describes the problem with the required assumptions, and presents the optimization problem formulation. The control policy structure is obtained numerically in section 4 . In section 5 , we present the simulation-based experimental approach used to determine the optimal lot sizing, the optimal hedging level and the optimal incurred cost. An illustrative numerical example is given and a sensitivity analysis is performed in order to confirm the robustness of the results. The paper is concluded in section 6 .

\section{Notations}

The following notations are used throughout the paper:

$\begin{array}{ll}x(.) & \text { Inventory level } \\ y(.) & \text { Inventory position } \\ u(.) & \text { Production rate (units/time) } \\ u^{i}(.) & \text { Production rate of the } i \text { th lot (units/time) } \\ u_{\max } & \text { Maximum production rate (units/time) } \\ d & \text { Constant demand rate (units/time) } \\ Q & \text { Lot sizing (units) } \\ q(.) & \text { Lot level at time } \mathrm{t} \text { (units) } \\ \theta_{i} & \text { Production start time of the } i \text { th lot } \\ \delta_{i} & \text { Production end time of the } i \text { th lot } \\ Q^{i} & \left.\text { ith lot to be produced during the interval }] \theta_{i}, \delta_{i}\right] \\ q_{\alpha \beta} & \text { Transition rate from mode } \alpha \text { to mode } \beta \\ M T B F & \text { Mean Time Between Failures } \\ M T T R & \text { Mean Time To Repair } \\ T T R_{k, i} & \text { kth repair time during the } i \text { th production run } \\ \tau & \text { Fixed transportation delay (time) } \\ c^{+} & \text {Unit holding cost (\$/unit) } \\ c^{-} & \text {Unit backlog cost }(\$ / \text { unit) } \\ c_{p} & \text { Unit production cost } \$ \text { /unit/time) } \\ c_{t} & \text { Fixed lot transportation }(\$ / \text { load) } \\ c_{v} & \text { Unit variable cost of transportation (\$/unit) } \\ \rho & \text { Discounted rate }\end{array}$




\section{Problem formulation}

\subsection{Problem description \& assumptions}

We study a single-product batch processing manufacturing system facing a high constant demand rate $d$. The system consists of a production facility subject to stochastic breakdowns and repairs, and supplying a downstream buffer stock $(B S)$ as illustrated in Figure 1. The $B S$ can be a local warehouse to serve the consumers directly, or an external warehouse to supply given retailers. Because the $B S$ is remote from the production facility, and to minimize the total transportation cost that depends essentially on the frequency of transportation and lot sizing, the production activities are planned in lots. The work-in-process (WIP) lot $q($.) is stored in a downstream area of the facility until the production lot is completed, after which it is transported to the $B S$. When the batch enters the $B S$, the inventory level $x($.) increases immediately with a finite jump equal to the lot sizing $Q$. The objective of the model is to jointly determine both the optimal lot sizing and the optimal control policy which minimize the total expected cost, including the WIP, the final inventory/backlog and the transportation costs.

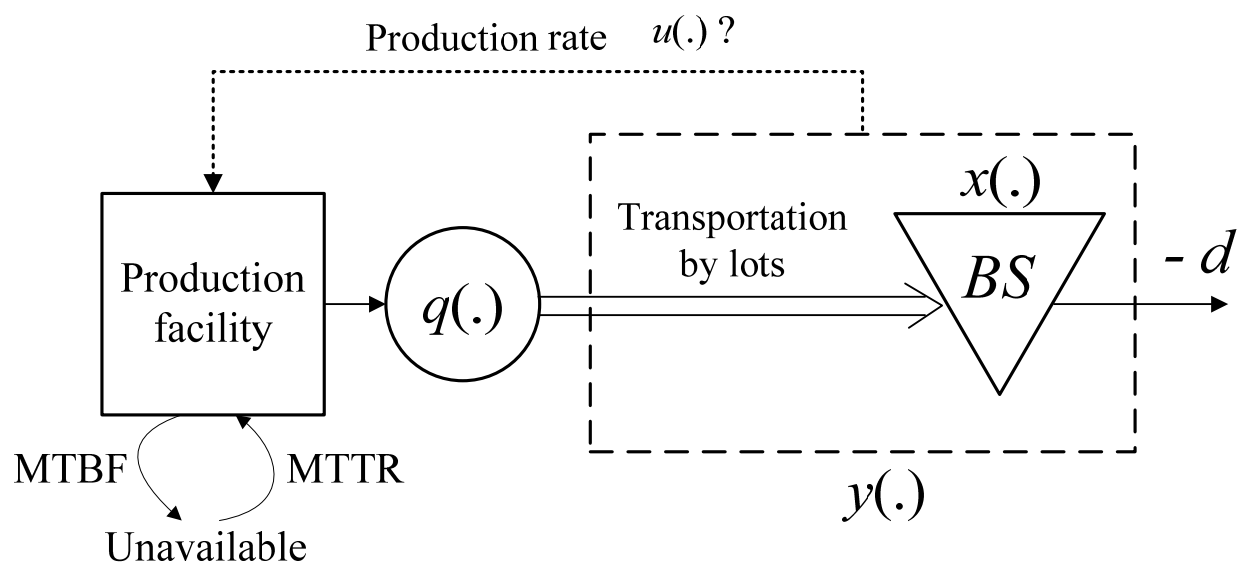

Figure 1. Unreliable manufacturing system with transportation delay.

The production run length of one lot is variable and depends on four factors: the production rate $u($.), the lot sizing $Q$, the random occurrence of failures and the random repair times. We describe an $i$ th production run by the interval $\left.] \theta_{i}, \delta_{i}\right]$. The production of the $i$ th lot starts at instant $\theta_{i}$, immediately after a production order is received, and finishes at instant $\delta_{i}$. The production end time $\delta_{i}$ can be written as follows: $\delta_{i}=\theta_{i}+Q^{i} / u^{i}()+.\sum_{k} T T R_{k, i}$, where $\sum_{k} T T R_{k, i}$ is the sum of repair times during the $i$ th production run. The $i$ th lot arrives at the final buffer stock at time 
$\left(\delta_{i}+\tau\right)$. Figure 2 presents a graphical description of the production dynamic $q($.$) , and final$ inventory level evolution $x($.$) in function of the instantaneous system availability and production$ runs lengths.

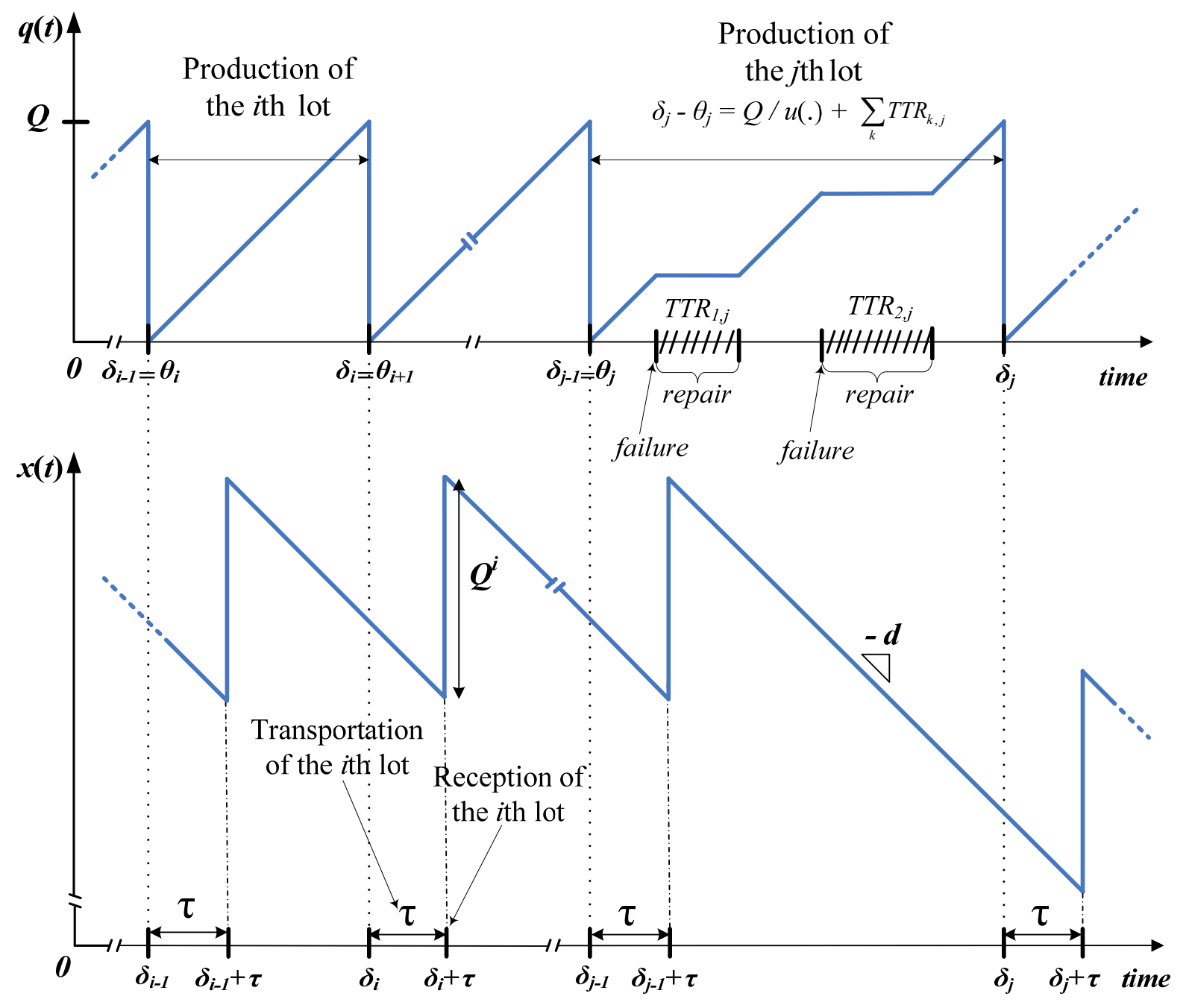

Figure 2. Production and final stock dynamics.

The following assumptions are made in formulating the problem:

- The time between the reception of the production order and the effective production run start is negligible.

- In the EMQ literature (e.g., Abboud (1997), Giri and Yun (2005), Sana and Chadhuri (2010)), there are some constraints concerning the number/distribution of failures occurrence in each production run. We extend our study to the general distribution of time between failures, and therefore to a no-limited number of failures during the production run.

- The production of interrupted lots is always resumed after repair. 
- The manufacturing system is perfect, and so the product quality is not affected by the reliability of the system.

- The transport means (trolley, truckload, etc.) are perfectly reliable and available to transport the lots immediately at the end of each production run. As in Li et al. (2009), the transportation delay is fixed and constant.

- The cost of one transportation operation is the sum of a fixed cost (since the transportation time is fixed), and a variable cost proportional to the lot sizing for loading and unloading operations.

- Shortages are allowed and penalized by a backlog cost.

\subsection{Optimization problem formulation}

The behaviour of the manufacturing system changes randomly with time. To formulate the dynamic and stochastic aspects of the considered problem, we have characterized the state of the manufacturing system at each instant $t$ with four components including:

- A piecewise continuous variable which describes the WIP level at time $t$ and denoted by $q(t)$. Let $0 \leq q(t) \leq Q^{i}, i=1 . . N$ be the capacity constraint of the WIP lot for each $i$ th production run.

- A piecewise continuous variable which describes the final buffer stock level at time $t$ and measured by $x(t)$. This quantity faces the continuous downstream demand $d$ and impulses upstream supply with finite jump $Q^{i}$ when an $i$ th lot produced is received from the production facility at instant $\delta_{i}+\tau$ after transportation.

- A piecewise continuous variable which describes the inventory position at time $t$ and is measured by $y(t), y(t)$ is equal to the instantaneous sum of the stock (inventory/backlog) level $x(t)$ and the total quantity of lots-under-transportation if existing. $\Rightarrow$ $y(t)=x(t)+\operatorname{Tr}(t) \cdot Q$, where $\operatorname{Tr}(t)$ is an integer variable instantaneously describing the total number of lots-under-transportation.

- A continuous stochastic process which describes the operational mode of the manufacturing system at time $t$, denoted by $\{\alpha(\mathrm{t})\}$ and taking values in $M=\{0,1\}$. $\alpha(t)=1 \Rightarrow$ The manufacturing system is operational $\alpha(t)=0 \Rightarrow$ The manufacturing 
system is down. Let $T=\left\{q_{\alpha \beta}\right\}$ be the transition rates matrix of the stochastic process $\{\alpha(\mathrm{t})\}$, with $q_{\alpha \beta} \geq 0$ if $\alpha \neq \beta$, and $q_{\alpha \alpha}=-q_{\alpha \beta(\alpha \neq \beta)}$, where $\alpha, \beta \in M$.

The dynamics of WIP lot level $q($.$) and final stock level x($.$) are given by the following$ differential equations:

$\left.\frac{d q(t)}{d t}=u(t, \alpha), q(0)=q_{0}, \forall t \in\right] \theta_{i}, \delta_{i}[$

$q\left(\delta_{i}^{+}\right)=q\left(\delta_{i}^{-}\right)-Q^{i}$

$\left.\frac{d x(t)}{d t}=-d, x(0)=x_{0}, \forall t \in\right] \delta_{i}+\tau, \delta_{i+1}+\tau[$,

$x\left(\left(\delta_{i}+\tau\right)^{+}\right)=x\left(\left(\delta_{i}+\tau\right)^{-}\right)+Q^{i}$,

$i=1, \ldots, N$

where $q$ and $x$ respectively denote the WIP level and the finished product stock level at the initial time. $\delta_{i}^{-}$and $\delta_{i}^{+}$denote the left and right boundaries of the $i$ th production run end time $\delta_{i}$ and, $\left(\delta_{i}+\tau\right)^{-}$and $\left(\delta_{i}+\tau\right)^{+}$denote the left and right boundaries of the $i$ th receipt time $\left(\delta_{i}+\tau\right)$.

At any given time, the production rate, the production order quantity and the WIP level must satisfy the production and system capacities constraints:

$0 \leq u(t, \alpha) \leq u_{\max }$

$0<Q^{i} \leq \min \left\{Q_{\max }^{w i p}, Q_{\max }^{t r}\right\}$

$0 \leq q(t, \alpha) \leq Q^{i}$

$i=1, \ldots, N$

where $Q_{\max }^{w i p}$ is the maximum WIP storage capacity, and $Q_{\max }^{t r}$ is the maximum transportation capacity.

Our decision variables are the production rate $u($.$) and a sequence of production orders denoted$ by $\Omega=\left\{\left(\theta_{1}, Q^{1}\right),\left(\theta_{2}, Q^{2}\right), \ldots,\left(\theta_{\infty}, Q^{\infty}\right)\right\}$ where, the couple $\left(\theta_{i}, Q^{i}\right)$ represents the production order of the $i$ th lot $Q^{i}$ at time $\theta_{i}$ (Figure 2). Let $\Gamma(\alpha)$ denote the set of admissible decisions $(\Omega, u()$. given by:

$$
\Gamma(\alpha)=\left\{(\Omega, u(.)) \mid 0 \leq u(t, \alpha) \leq u_{\max }, 0<Q^{i} \leq \min \left\{Q_{\max }^{w i p}, Q_{\max }^{t r}\right\}\right\}
$$


The instantaneous cost function $g($.$) including the production, the WIP stocking, and the finished$ product inventory and backlog costs is given by the following equation:

$$
\left.g(q(t), x(t), u(t, \alpha))=c^{+}\left(q(t)+T_{r}(t) Q+x^{+}(t)\right)+c^{-} x^{-}(t)+c_{p} u(t), \forall t \in\right] \delta_{i}, \delta_{i+1}[
$$

where, $x^{+}(t)=\max (0, x(t))$ and $x^{-}(t)=\max (0,-x(t))$.

In addition, the instantaneous cost function of the transportation at times $\delta_{i}(i=1, \ldots, N)$, is given by the following equation:

$$
R\left(Q^{i}, \alpha\right)=\operatorname{Ind}\left(t=\delta_{i}\right)\left(c_{t}+c_{v} Q^{i}\right)+\int_{0}^{\tau} e^{-\rho \cdot t} g(q, x-d . t, u) d t
$$

where, Ind $(\Theta())=.\left\{\begin{array}{ll}1 & \text { if } \Theta(.) \text { is true } \\ 0 & \text { otherwise }\end{array}\right.$.

Using (3) and (4), the overall infinite horizon discount cost $J($.$) is defined as follows:$

$$
J(q, x, \Omega, u, \alpha)=E\left\{\int_{0}^{\infty} e^{-\rho t} g(q, x, u) d t+\sum_{i=0}^{\infty} e^{-\rho \delta_{i}}\left(c_{t}+c_{v} Q\right) \mid q(0)=q_{0}, x(0)=x_{0}, \alpha(0)=\alpha\right\}
$$

where, $E\{$.$\} is the expectation operator conditioned on the initial conditions (q, x, \alpha)$.

The production planning problem considered here involves seeking an admissible control policy $\left(\Omega^{*}, u^{*}\right)$ that minimizes $J($.$) given by (5) considering equations (1) to (4). This is a feedback$ control policy that determines the production orders and the production rate as a function of the $\operatorname{system} \operatorname{state}(q, x, \alpha)$.

The value function $v($.) associated with such a stochastic optimal control problem is given by:

$$
v(q, x, \alpha)=\inf _{(\Omega, u) \in \Gamma(\alpha)} J(q, x, \Omega, u, \alpha), \quad \forall \alpha \in M
$$

As in Sethi and Zhang (1994), and using the optimal impulsive control theory (Sethi and Thompson, 2005), it can be shown that the value function $v(q, x, \alpha)$ is the unique viscosity solution for the following HJB equations : 


$$
\min \left\{\begin{array}{c}
\min _{u}\left\{(u) v_{q}+(-d) v_{x}+g(q, x, u)+\sum_{\alpha \neq \beta} q_{\alpha \beta}(v(q, x, \alpha)-v(q, x, \beta))\right\}-\rho v(q, x, \alpha) ; \\
\min _{Q}\left\{R(Q, \alpha)+e^{-\rho \tau} v(q-Q+\tau d, x+Q-\tau d, \alpha)\right\}-v(q, x, \alpha)
\end{array}\right\}=0
$$

where, $v_{q}$ and $v_{x}$ respectively denotes the gradients of $v($.$) with respect to q$ and $x$. The details of optimality conditions given by (7) and elementary properties of the value function can be found in Hajji et al. (2009).

The control policy $\left(\Omega^{*}, u^{*}\right)$ that we are seeking can be obtained when the value function is known. Given the difficulty of solving the equation (7) analytically, we advocate a numerical approach to obtain an approximation of the value function and the associated control policy.

\section{Approximated structure of the optimal control policy}

\subsection{Numerical resolution approach}

To facilitate the numerical approach implementation process, the resolution is applied in this section to a negligible delay problem $(\tau \approx 0)$. That means that, once produced, the lot immediately supplies the final stock. Thus, the HJB equations become:

$$
\min \left\{\begin{array}{c}
\min _{u}\left\{(u) v_{q}+(-d) v_{x}+g(q, x, u)+\sum_{\alpha \neq \beta} q_{\alpha \beta}(v(q, x, \alpha)-v(q, x, \beta))\right\}-\rho v(q, x, \alpha) ; \\
\min _{Q}\{R(Q, \alpha)+v(q-Q, x+Q, \alpha)\}-v(q, x, \alpha)
\end{array}\right\}=0
$$

The numerical method applied here to determine the structure of the optimal control policy is based on the Kushner approach (Kushner and Dupuis, 1992). This approach consists in iteratively approximating the value function $v(q, x, \alpha)$ and its derivative for discrete values of the state variables $(q, x, \alpha)$. The implementation of the approximation technique requires the use of a finite grid denoted $D_{h}$ here, where $h(h>0)$ is a given vector of finite difference intervals. The computation domain $D_{h}$ is defined as follow:

$$
D_{h}=\{(q, x): 0 \leq q \leq a,-b \leq x \leq b\}
$$

where $a$ and $b$ are given positive constants.

Then, the value function $v(q, x, \alpha)$ is approximated by $v^{h}(q, x, \alpha)$ and their partial derivatives are approximated by: 


$$
\begin{aligned}
& (v)_{q}(q, x, \alpha)=\frac{1}{h}\left(v^{h}(q+h, x, \alpha)-v^{h}(q, x, \alpha)\right) \\
& (v)_{x}(q, x, \alpha)=\frac{1}{h}\left(v^{h}(q, x, \alpha)-v^{h}(q, x-h, \alpha)\right)
\end{aligned}
$$

The approximated value function $v^{h}(q, x, \alpha)$ is obtained by solving the modified HJB equations with the appropriate boundary conditions (Kenné et al., 2003).

For a given finite difference interval $h$, the iterative approximation algorithm is given by the following six steps:

Step 1. Initialization. Choose a precision $\gamma \in R^{+}$. Set $\mathrm{n}:=1$, and $\left(v^{h}(q, x, \alpha)\right)^{n}:=0, \forall \alpha \in M$, $\forall q, x \in D_{h}$.

Step 2. Set $\left(v^{h}(q, x, \alpha)\right)^{n-1}:=\left(v^{h}(q, x, \alpha)\right)^{n}, \forall \alpha \in M, \forall q, x \in D_{h}$.

Step 3. Compute the corresponding cost function from the first part of (8). Obtain $u^{n}($.$) .$

Step 4. Compute the corresponding cost function from the second part of (8). Obtain $Q^{n}($.$) .$

Step 5. Compute the corresponding cost function from (8). Obtain $u^{n}($.$) and Q^{n}$ (.) for the given value function, $\forall \alpha \in M, \forall x \in D_{h}$.

Step 6. Stop test.

$$
\begin{aligned}
& \bar{c}:=\min _{q, x \in D_{h}}\left[\left(v^{h}(q, x, \alpha)\right)^{n}-\left(v^{h}(q, x, \alpha)\right)^{n-1}\right] \\
& \underline{c}:=\max _{q, x \in D_{h}}\left[\left(v^{h}(q, x, \alpha)\right)^{n}-\left(v^{h}(q, x, \alpha)\right)^{n-1}\right] \\
& c_{\min }:=\frac{\rho}{1-\rho} \bar{c} \quad c_{\max }:=\frac{\rho}{1-\rho} c \\
& \text { if }\left|c_{\max }-c_{\min }\right| \leq \gamma \text { then stop, }\left(u^{*}, Q^{*}\right):=\left(u^{n}, Q^{n}\right) \\
& \text { else } n:=n+1 \text { and go to step 2. }
\end{aligned}
$$

\subsection{Numerical example}

In this section, we present a numerical example for the case of negligible transportation delay. The example parameters values are as follows: $u_{\max }=150, d=120, \rho=0.1, q_{01}=0.1, q_{10}=0.01$, $c^{+}=10, c^{-}=150, c_{t}=50, c_{v}=1, c_{p}=1$. The computational domain $D_{h}$ given by (9) is taken for $a=100$ and $b=500$ with $h=10$. 
To ensure a clear characterization of the control policy, several elements are taken into consideration as part of the implementation process. Indeed, the production and lot sizing policies are each observed separately. For each policy, the relevant significant stock threshes are analyzed independently of the others. For each numerical result, $U(q, x, 1)$ and $Q(q, x, 1)$ are the production policy and the lot sizing policy function of the state variables respectively, as shown in the following figures. The numerical results for the basic case study are shown in Figures 3 and 4.

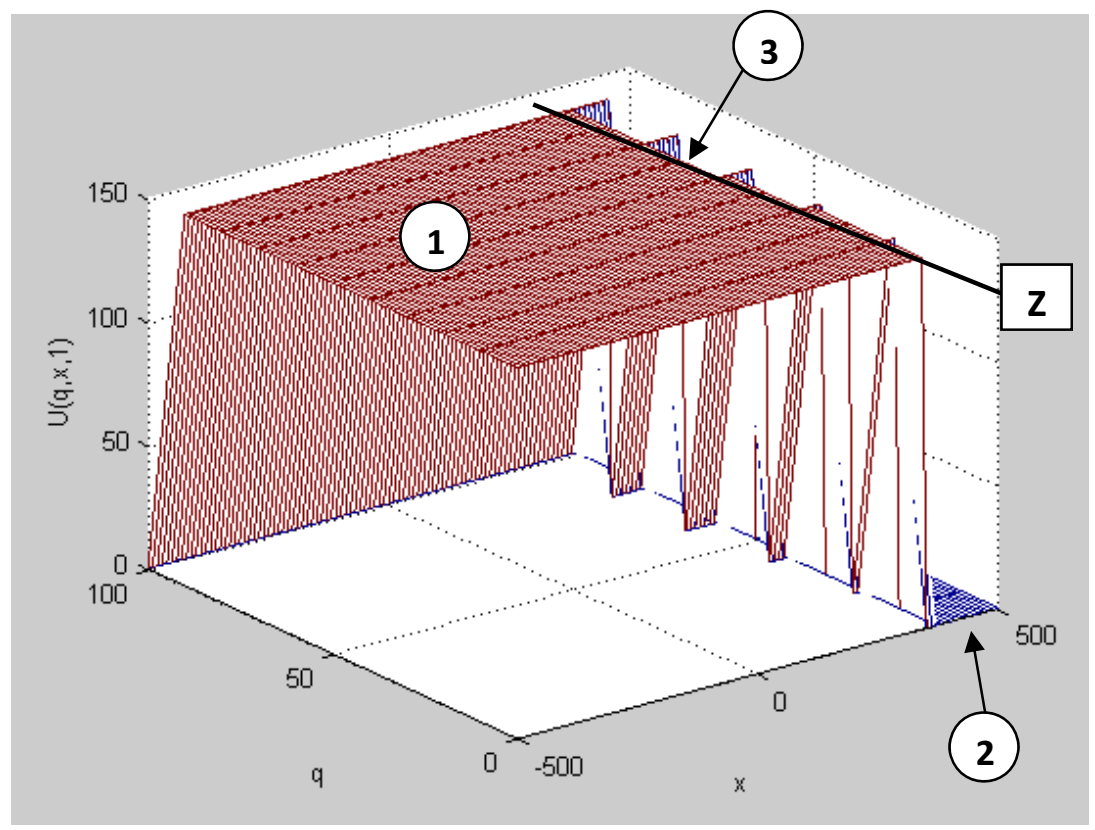

Figure 3. Production control policy

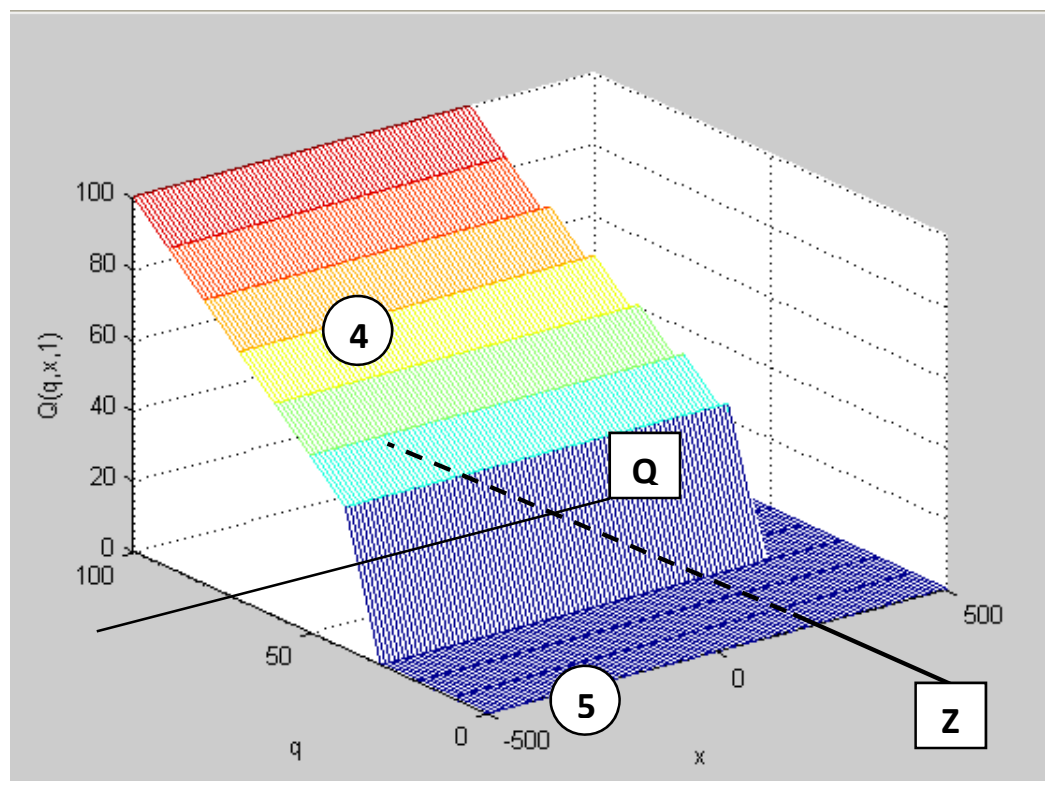

Figure 4. Lot sizing control policy 
It follows from our numerical results that the resulting production policy divides the surplus space into two mutually exclusive regions. In region (1), produce at the maximal rate, in region (2) set the production rate equal to zero and between regions (1) and (2) (region (3), set the production rate equal to the demand rate. These results point towards a Base Stock Policy (BSP for short) type of production control, given that, at any time, the production rate is either at the lowest, demand or maximum level depending on the inventory level $x$. The BSP is governed by the threshold parameter $Z$ as shown in Figure 3 .

Furthermore, the results show that the lot sizing policy is governed by a State Dependent Economic Manufacturing Quantity policy, SD-EMQ for short. This policy divides the surplus space into two mutually exclusive regions: region (4), is governed by an Economic manufacturing quantity ( $Q$ as shown in Figure 4$)$ to be processed if the batch quantity is available in the stock

area $q$ and the finished product stock level $x$ is lower than a threshold level $Z$, and in region (5), no batch is transferred to the finished stock area $(Q=0)$. Note that the numerical results show that the threshold $\mathrm{Z}$ characterizing the production control policy is exactly equal to that characterizing the lot sizing control policy. To ensure that the structure of the optimal policy, governed by the threshold parameter $Z$ and the economic lot $Q$, is always maintained, a sensitivity analysis was conducted. The results obtained confirmed our expectations and allowed us to conclude that the optimal policy is a combination of BSP and SD-EMQ policies.

\subsection{Control policy structure}

According to the numerical results presented in the previous section, the optimal control policy in the case of a negligible transportation delay can be approximated by a combination of BSP and SD-EMQ policies, which can be expressed by the following equations:

Production order policy;

$\Omega^{i}\left(t \geq \delta_{i-1}, Q\right)=\left\{\begin{array}{cc}Q^{i} & \text { if }\left(x\left(\delta_{i-1}^{+}\right) \leq Z\right) \operatorname{or}\left((x(t)=Z) \mid\left(x\left(\delta_{i-1}^{+}\right)>Z\right)\right) \\ 0 & \text { Otherwise }\end{array}, i=1,2, . ., \infty\right.$

Production-inventory control policy; 
$\left.\left.u^{i}(t \in] \theta_{i}, \theta_{i+1}\right], \alpha\right)= \begin{cases}u_{\max } & \text { if }\left(x\left(\theta_{i}^{+}\right)<Z\right) \&\left(\theta_{i}<t \leq \delta_{i}\right) \&(\alpha(t)=1) \\ d & \text { if }\left(x\left(\theta_{i}^{+}\right)=Z\right) \&\left(\theta_{i}<t \leq \delta_{i}\right) \&(\alpha(t)=1) \quad, i=1,2, . ., \infty \\ 0 & \text { if }\left\langle\left(x\left(\delta_{i}^{+}\right)>Z\right) \&\left(\delta_{i}<t \leq \theta_{i+1}\right)\right\rangle \operatorname{or}(\alpha(t)=0)\end{cases}$

with these following constraints:

$0<Q \leq \min \left\{Q_{\max }^{\text {wip }}, Q_{\max }^{t r}\right\}, Z \geq 0$.

To consider the transportation delay on the control policy, we propose some adjustments. In the literature, as in Mourani et al. (2008) and Li et al. (2009), the production control policies with considerable transportation delay are based on controlling the inventory position which takes into account the on-hand inventory and the total quantity of lots-under-transportation. The relationship between the inventory level $x(t)$, the inventory position $y(t)$ and the demand during the transportation delay $\tau \cdot d$ can be written as follows (Mourani et al., 2008):

$$
x(t)=y(t-\tau)-\tau \cdot d, \forall t \geq \tau
$$

Note that when the transportation delay is negligible, the inventory position $y(t)$ coincides with the inventory level $x(t)$. Then, we extend the control policy in the case of non-negligible transportation delay using the concept of the inventory position as follows:

\section{Production order policy;}

$\Omega^{i}\left(t \geq \delta_{i-1}, Q\right)=\left\{\begin{array}{cc}Q^{i} & \text { if }\left(y\left(\delta_{i-1}^{+}\right) \leq Z\right) \operatorname{or}\left((y(t)=Z) \mid\left(y\left(\delta_{i-1}^{+}\right)>Z\right)\right) \\ 0 & \text { Otherwise }\end{array}, i=1,2, . ., \infty\right.$

Production-inventory control policy;

$\left.\left.u^{i}(t \in] \theta_{i}, \theta_{i+1}\right], \alpha\right)= \begin{cases}u_{\max } & \text { if }\left(y\left(\theta_{i}^{+}\right)<Z\right) \&\left(\theta_{i}<t \leq \delta_{i}\right) \&(\alpha(t)=1) \\ d & \text { if }\left(y\left(\theta_{i}^{+}\right)=Z\right) \&\left(\theta_{i}<t \leq \delta_{i}\right) \&(\alpha(t)=1) \quad, i=1,2, . ., \infty \\ 0 & \text { if }\left\langle\left(y\left(\delta_{i}^{+}\right)>Z\right) \&\left(\delta_{i}<t \leq \theta_{i+1}\right)\right\rangle \operatorname{or}(\alpha(t)=0)\end{cases}$

with the following constraints:

$0<Q \leq \min \left\{Q_{\max }^{\text {wip }}, Q_{\max }^{\text {tr }}\right\}, Z \geq 0$.

The production order is controlled by the equation (13). (i) If at the end of the (i-1)th production run $\left(t=\delta_{i-1}^{+}\right)$, the inventory position is less than or equal to the threshold $Z$, a new production 
order $Q^{i}$ is launched (Areas $(1)$ and $(3$ - Figure 5). In this case, the start time of the $i$ th production run coincides exactly with the end time of the (i-1)th production run $\Rightarrow \delta_{i-1}^{+}=\theta_{i}$. (ii) If at the end of the $(i-1)$ th production run, the inventory position is greater than the threshold $Z$, the production is stopped until the inventory level decreases to the level $Z$ (Area ${ }^{2}$ - Figure 5). At that time, a new production order is placed to produce the $i$ th lot $Q^{i}$. The production of the lot starts immediately and we mark this time by $\theta_{i}$. The relationship between the production end time of an $(i-1)$ th lot and the production start time of a next $i$ th lot can be expressed as follows : $\theta_{i}=\delta_{i-1}+\operatorname{Ind}\left(y\left(\delta_{i-1}^{+}\right)>Z\right) \cdot \frac{y\left(\delta_{i-1}^{+}\right)-Z}{d}$. The next $(i+1)$ th production order control $\Omega^{i+1}($.$) is$ done at time $\delta_{i+1}^{+}$. (iii) Beyond these two cases, no production order is transmitted (Area ${ }^{4}$ Figure 5).

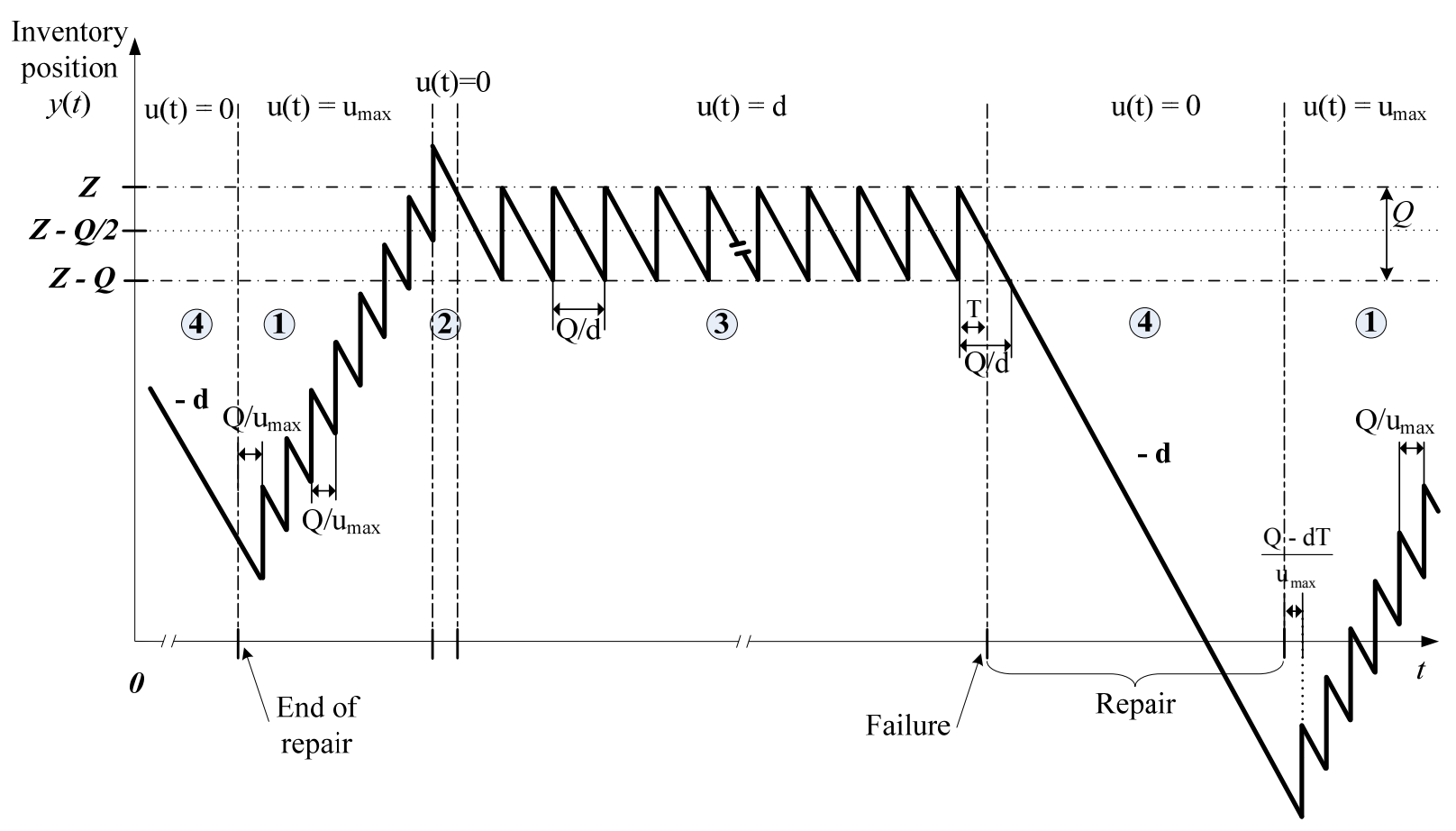

Figure 5. Structure of the production control policy

The production-inventory policy is controlled by equation (14). Note that the feedback control policy has a structure similar to the so-called hedging point policy. The production rate has three possible levels: $u_{\max }$ (accelerated production), $d$ (normal production), and 0 (no production). The feedback policy consists in controlling the inventory level at the beginning and at the end of each production run, and instantly controlling the system availability in order to determine the 
corresponding production rate. We define the threshold $Z$ as the hedging level. (a) If the inventory position at the moment of releasing an $i$ th production order (at the beginning of the production of the $i$ th lot) is strictly below the hedging threshold $Z$, the lot is produced at the maximum production rate $u_{\max }$. This is the case when the production is restarting just after a corrective maintenance. At the end of each last repair, the lot produced is added to the inventory position after a delay $Q / u_{\max }($ Area 1 - Figure 5). (b) Because the inventory position increases with a finite impulsive jump when a lot produced is moved to the final stock, the inventory position could very well exceed the threshold $Z$ after each production restart. In this case, the production is stopped $(u()=0$.$) until the inventory position falls to the threshold Z$ as a result of the demand (Area 2 - Figure 5). (c) At that point, the production is resumed with a production rate equal to the demand rate $(u()=d$.$) . Thus, the production run length is Q / d$. Since the demand rate is constant, the inventory position is equal to $Z-Q$ at the end of the production run. Given that the lot is transported to the final stock immediately after the end of the production run, the inventory level rises back to level $Z$. While the production system is operational, the inventory level continues its variation between levels $Z$ and $Z-Q$, as shown in Area 3 - Figure 5 .

Because the production control policy is the structured as the HPP, and as the manufacturing quantity $Q$ is a decision variable that should be optimized, we call the obtained control policy EMQ-HPP. This policy is completely defined for given values of $Q$ and $Z$, which here, are called design factors.

Although the numerical resolution provides the structure of a near-optimal control policy, implementation difficulties and irregularities in the boundary of the numerical results render the approximation of the control parameters challenging. Furthemore, the accuracy of the value function and of the related control parameters obtained through the numerical approach depends on the fineness of the grid step (Kenné et al., 2003). A satisfactory approximation would be too time-consuming to be applicable at the operational level. In the next section, we propose an alternative approach based on simulation in order to determine a very close approximation of the optimal control policy parameters. Moreover, the use of simulation allows an extension of application of the control policy to general failure and repair distributions. 


\section{Estimation of the optimal control policy}

\subsection{Control approach}

To determine the optimal values of the design factors $\left(Q^{*}, Z^{*}\right)$ of the EMQ-HPP, we adopt an experimental-control approach based on Kenné and Gharbi (1999, 2000). This approach combines both analytical and simulation modeling, experimental design and response surface methodology. It our study, it can be applied through the following six steps:

- Step 1. Batch production control policy $\left(u^{*}, Q^{*}\right)$ ? : This step specifies the objective of the study, as described in the section 3.1. The objective here is to find the optimal manufacturing control variables: the optimal production rate control $u($.$) and the optimal lot \operatorname{sizing} Q$, that minimize the total incurred cost of inventory/backlog and transportation.

- Step 2. Analytical model and optimization problem formulation: The objective of this step is to formulate the problem as a stochastic dynamic programming model and to obtain the HJB equations (section 3.2).

- Step 3. Structure of the optimal control policy: Using the numerical method, the structure of the optimal control policy is approximated and characterized by the design factors $(Q, Z)$ (section 4). The sub-optimal control policy with a considerable transportation delay is refined in section 5.1.

- Step 4. Simulation model: The simulation model describes the dynamic of the system using the control policy obtained, and evaluates its performances for given factors $(Q, Z)$. These factors are considered as input of such a model, and the related incurred cost is defined as its output. For more details concerning the simulation model, the reader is referred to the next section.

- Step 5. Experiment design: The experimental design defines how the control factors $(Q, Z)$ should be varied in order to determine the effects of the mains factors and their interactions (i.e., ANOVA analysis of variance) on the incurred total cost through a minimal set of simulation runs.

- Step 6. Response surface methodology: The main significant factors and their interactions are considered as the input of a regression analysis, used in conjunction with the response surface methodology, to fit the relationship between the cost and the input factors. The regression 
model obtained is then optimized to determine the optimal values of the EMQ-HPP parameters $\left(Q^{*}, Z^{*}\right)$ and the optimal total expected cost.

\subsection{Simulation model}

A combined discrete-continuous model was developed using the SIMAN simulation language with a $\mathrm{C}++$ subroutine (Pegden et al., 1995), and then executed through the ARENA simulation software. The model consists of several networks describing specific tasks and events in the system. Figure 6 presents a simple block-diagram schema of the simulation model. We can see three principal networks described as follows: The network $(I)$ is developed as a discrete submodel in order to model the production and the transportation operations. The inventory position is controlled at the beginning of each production run to determine the corresponding production rate as described in equation (14). The entity here represents the lot to be produced. This network is connected with the $\mathrm{C}++$ routine (II), which instantaneously calculates the WIP and the inventory levels using the differential equations of (1). The $q($.$) and x($.$) variables are$ integrated using the Runge-Kutta-Fehlberg (RKF) method which guarantees a consistent accuracy as mentioned in Pegden et al. (1995). When a lot produced is released or a transported lot is transferred to the final stock, the WIP and inventory levels are updated with the corresponding impulse equation of (1). Furthermore, the $\mathrm{C}++$ routine allows the surplus/backlog level to be calculated each time. The discrete network (III) models the failure and repair events. The time between failures and the time of repair are determined randomly from the MTBF and MTTR distributions. Note that the model is developed to accept any probability distribution for the MTBF and the MTTF. The network (III) is connected to the $\mathrm{C}++$ routine in order to integrate the availability state of the manufacturing system in the computation of the WIP level. It should be recalled that when the system fails, the production is immediately stopped and therefore the WIP level $q($.) is maintained constant until the production is resumed after a system repair. 


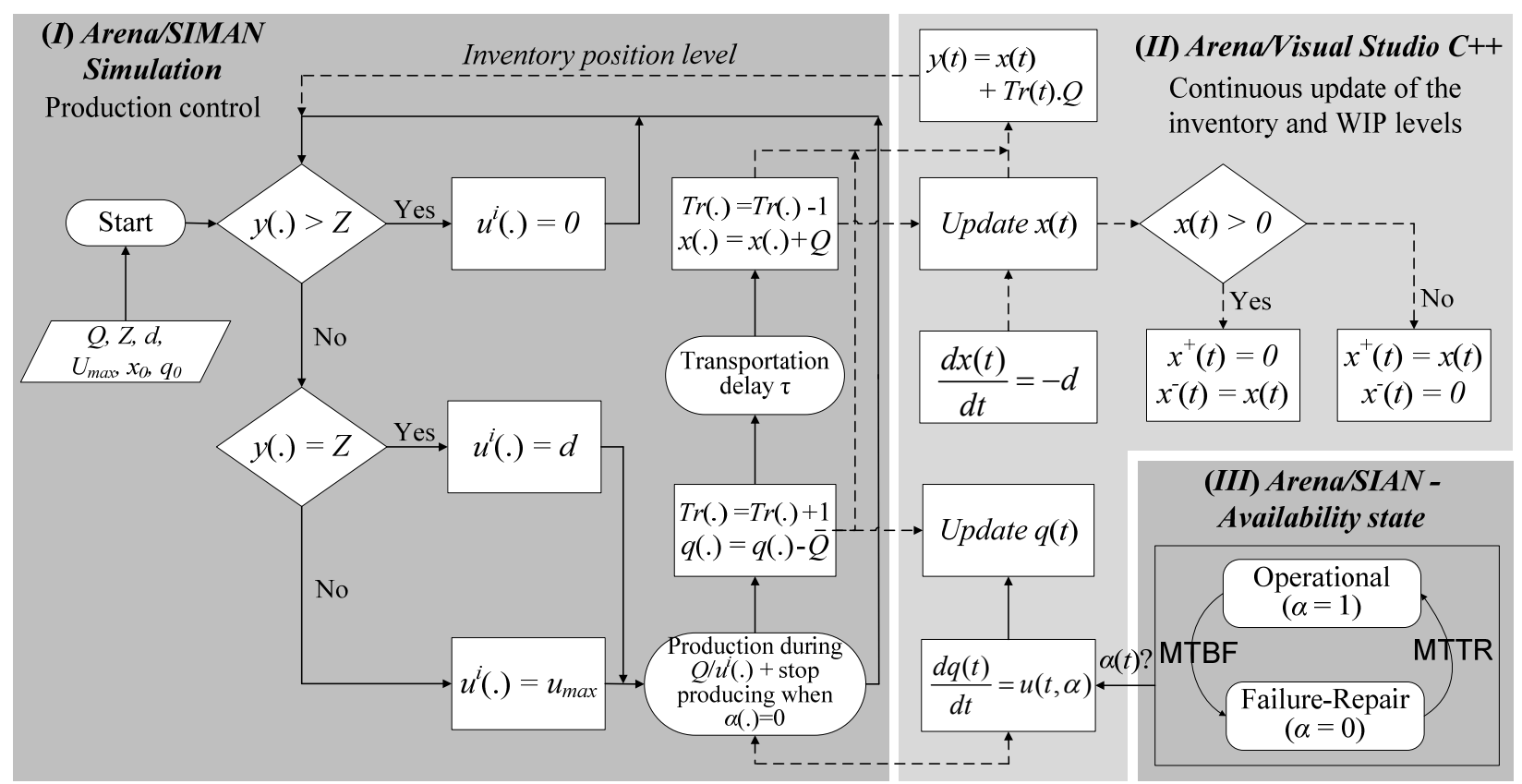

Figure 6. Block-diagram representation of the simulation model.

\subsection{Verification and validation of the simulation model}

To verify and validate the accuracy of the simulation model, we graphically examine its behaviour. Figure 7 shows the trajectories of the production rate and the inventory level and position, for a sample of the simulation run. The graphic shows that the model performs correctly according to the logic of the production-inventory control policy. The production rate value changes instantaneously in response to changes in the inventory position and the system availability state as described in equation (14). We also verify that, during the periods of normal production $(u()=d$.$) , the inventory position varies between the hedging level Z$ and the $Z-Q$ level as explained in section 4.3. The impact of the transportation delay is clearly shown on the time lag of the inventory level trajectory, as compared with the inventory position trajectory as described in equation (14). The model has also been validated in the case where the lot sizing is set to value 1 (item-by-item production) and the transportation delay is negligible, with Kenné and Gharbi (2000) model. 


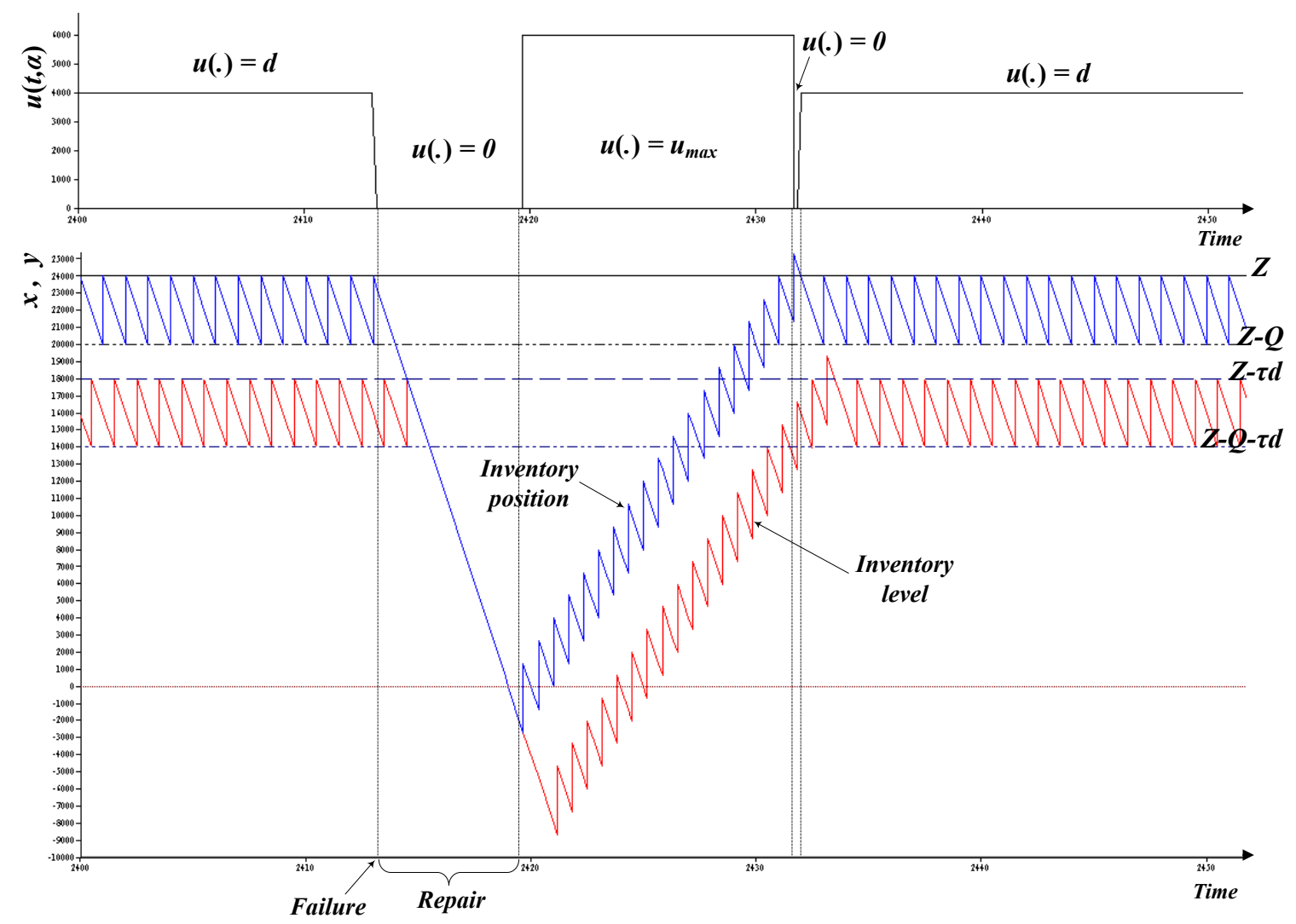

Figure 7. Inventory level/position dynamic.

\subsection{Experimental design, ANOVA and response surface methodology}

Two independent variables $(Q, Z)$ and one dependent variable (the total expected cost) are considered. Because of the convexity property of the value function (6) at the inventory level $x$ (Sethi and Zhang, 1994), a quadratic model is considered to fit the cost function. The idea of approximating the function cost using a quadratic model has been used in the literature (see Ryzin et al. (1991), Gershwin (1994), Kenné and Gharbi (1999)). A complete statistical analysis is applied to obtain a very close approximation of the value function, as follows:

1. A complete $3^{2}$ experimental design is selected and five replications are conducted for each combination of factors $(Q, Z)$. This requires $45\left(3^{2} \times 5\right)$ simulation runs. To reduce the variability in the simulation results from one configuration to another, we use the common random number technique (Kelton and Law, 2000). Then, the experimental design is used to study and understand the effects of the input factors on the performance measure (i.e., the cost). 
2. A multi-factor statistical analysis (ANOVA) of the simulated data is carried out using statistical software (STATISTICA) to provide the effects of the design factors, their interaction and their quadratic effects on the response variable (i.e., the cost).

3. A response surface methodology is applied in order to optimize the response variable as a function of the significant effects. We assume that a continuous function $\Phi($.) exists, fitting a second-order regression model relating the response variable to the design factors. The function $\Phi($.$) is called the response surface and takes the following equation:$

$$
\Phi(Q, Z)=\beta_{0}+\beta_{1} Q+\beta_{2} Z+\beta_{12} Q Z+\beta_{11} Q^{2}+\beta_{22} Z^{2}+\varepsilon
$$

where, $\beta_{0}, \beta_{i}(\mathrm{i}=1,2), \beta_{12}, \beta_{i i}(\mathrm{i}=1,2)$ are unknown parameters to be estimated from the collected simulation data, and $\varepsilon$ is a random error. For more details on the statistical analysis, the reader is referred to Montgomery (2008).

\subsection{Numerical example}

To illustrate the simulation-based experimental approach, we perform a step-by-step determination of the optimal design factors $\left(Q^{*}, Z^{*}\right)$ of the control policy for a basic numerical example. The example data parameters are as follows: $u_{\max }=6000, d=4000, \tau=3$, $M T B F \square \log -\operatorname{Normal}(50,5), M T T F \square \operatorname{Gamma}(0.5,10), c^{+}=0.1, c^{-}=1.0, c_{t}=1200, c_{v}=0.01$, $c_{p}=0.25$. The values of the maximum production rate $u_{\max }$ and the demand rate $d$ are chosen such as to respect the feasibility condition of the system described by this inequality: $\frac{M T B F}{M T B F+M T T R} u_{\max }>d$.

To ensure that the steady-state is reached, the duration of simulation run is set such as to observe 10,000 failures in each replication, i.e., at 500,000 units of time. Simulation runs are conducted according to the experiments design presented in the previous section.

Table 1 summarizes the ANOVA of the collected data. For each design factor (including the linear and the quadratic effect) and its interaction, the table presents the sum of squares (SS), the degree of freedom (Df), the mean square (MS), an F-ratio, all computed using the residual mean square, and the significance level of the P-value. 
Table 1. ANOVA table for the total cost.

\begin{tabular}{lrrrrrr}
\hline Factor & SS & Df & MS & F-Ratio & P-value & Significant \\
\hline$Q$ (Linear + quadratic) & 82506.8 & 2 & 41253.4 & 238.1415 & 0.000000 & $\mathrm{~S}$ \\
$Z$ (Linear + quadratic) & 106147.9 & 2 & 53074.0 & 306.3776 & 0.000000 & $\mathrm{~S}$ \\
$Q . Z$ & 168711.1 & 1 & 168711.1 & 973.9107 & 0.000000 & $\mathrm{~S}$ \\
Blocks & 336.4 & 4 & 84.1 & 0.4855 & 0.746215 & $\mathrm{NS}$ \\
Error & 6063.1 & 35 & 173.2 & & & \\
Total SS & 363765.3 & 44 & & & & \\
\hline $\mathrm{R}^{2}=0.9833, \mathrm{R}^{2}$ (adjusted) $=0.9790$ & & & & &
\end{tabular}

The linear and quadratic effects of the two factors $(Q, Z)$ and their interaction $Q . Z$ are significant for the dependent variable (symbol $\mathrm{S}$ in the last column), at a 0.05 level of significance. The Rsquared adjusted value of 0.9790 presented in Table 1, states that $97.90 \%$ of the observed variability in the total expected cost is explained by the model (Montgomery, 2008). A residual analysis was also used to verify the adequacy of the model. A residual versus fitted values plot and a normal probability plot of residuals were used to test the homogeneity of the variances and the residual normality, respectively. Therefore, it was confirmed that the total expected cost can be fitted by a quadratic model as written in equation (15). From STATISTICA, the corresponding second-order model is given by:

$$
\begin{aligned}
\Phi(Q, Z)= & 9321.55+63.37 \times 10^{-3} Q-295.70 \times 10^{-3} Z-7.65 \times 10^{-6} Q Z \\
& +9.89 \times 10^{-6} Q^{2}+5.80 \times 10^{-6} Z^{2}+\varepsilon
\end{aligned}
$$

Figure 8 presents the projection of the cost response surface (16) on a two-dimensional plan $(Q$, $Z$ ). The minimum total expected cost 4961.68 is located at $Q^{*}=8944$ and $Z^{*}=31405$ as shown in Figure 8 . These values constitute the optimal parameters of the sub-optimal control policy which should be applied to control the production orders and the production rate of the manufacturing system as defined in equations (13) and (14). 


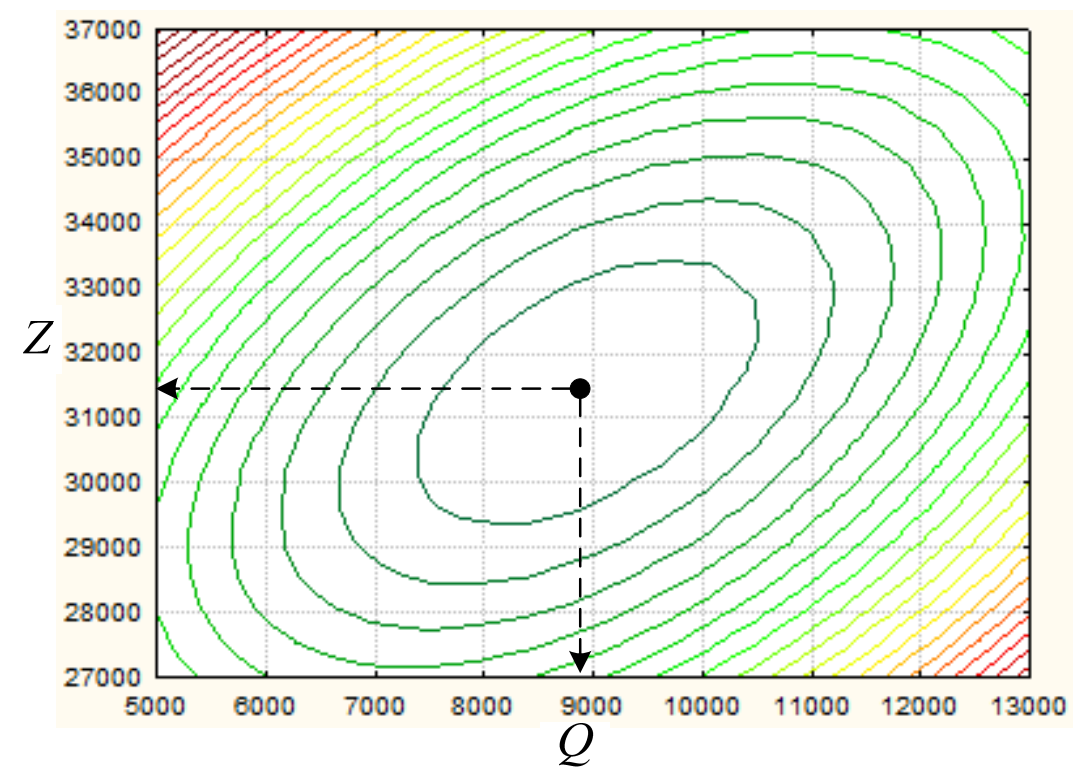

Figure 8. Cost response surface.

\subsection{Sensitivity analysis of cost parameters}

A sensitivity analysis of the control policy is conducted with respect to inventory, backlog and transportation costs (i.e., $c^{+}, c^{-}$and $c_{t}$ ). The objective is to prove the efficiency and the robustness of the experimental resolution approach applied to the adjusted EMQ-HPP policy. For twelve cases of costs changes derived from the basic case, the optimal design factors and incurred cost variations (i.e., respectively $\Delta \mathrm{Q}^{*}, \Delta \mathrm{Z}^{*}$ and $\Delta \mathrm{C}^{*}$ ) are explored and discussed. The results are summarized in Table 2.

- Variation of the inventory cost: When the inventory cost is higher (cases 3-4), the optimal hedging level $Z^{*}$ decreases in order to avoid further inventory costs. The optimal lot sizing $Q^{*}$ decreases to reduce the WIP holding cost, and to ensure a better supply to the final stock against the risk of shortages becoming higher. Conversely (cases 1-2), the stock level increases in order to further avoid increasing the backlog costs. Since the average stock level is more comfortable than that of the basic case, the optimal lot sizing increases in order to reduce transportation costs. Note that, with the condition $Q<c_{t} / c_{v}$, the increase in the lot sizing leads to a decrease in the transportation frequency, and hence, a decrease in the total transportation cost. 
Table 2. Sensitivity analysis for different cost parameters.

\begin{tabular}{|c|c|c|c|c|c|}
\hline \multirow{2}{*}{ Case } & \multicolumn{2}{|c|}{ Costs changes } & \multicolumn{2}{|c|}{ Optimal parameters variations } & \multirow{2}{*}{$\Delta \mathrm{C}^{\prime}$} \\
\hline & Costs & Changes $(\%)$ & $\Delta Q^{*}$ & $\Delta Z^{*}$ & \\
\hline 1 & $c^{+}$ & $-50 \%$ & +2115 & +5651 & -1541.59 \\
\hline 2 & & $-25 \%$ & +1059 & +2825 & -736.32 \\
\hline 3 & & $+25 \%$ & -1063 & -2823 & +667.44 \\
\hline 4 & & $+50 \%$ & -2130 & -5646 & +1266.03 \\
\hline 5 & $c^{-}$ & $-50 \%$ & +200 & -8275 & -506.08 \\
\hline 6 & & $-25 \%$ & +115 & -2746 & -184.2 \\
\hline 7 & & $+25 \%$ & -128 & +1618 & +128.28 \\
\hline 8 & & $+50 \%$ & -262 & +2669 & +228.26 \\
\hline 9 & $c_{t}$ & $-50 \%$ & -4604 & -3039 & -346.25 \\
\hline 10 & & $-25 \%$ & -1529 & -1009 & -147.18 \\
\hline 11 & & $+25 \%$ & +915 & +604 & +126.56 \\
\hline 12 & & $+50 \%$ & +1524 & +1006 & +242.84 \\
\hline
\end{tabular}

- Variation of backlog cost: When the backlog cost increases (cases 7-8), a higher stock level must be held in order to better protect the system against shortages, which explains the increase in the optimal hedging level $Z^{*}$. The optimal lot sizing $Q^{*}$ decreases in order to reduce the production delay, and therefore ensure better supply to the final stock against the risk of shortages. The decrease in backlog cost (cases 5-6) produces the opposite effects.

- Variation of transportation cost: When the fixed transportation cost $c_{t}$ is higher (cases 11-12), the system reacts by reducing the frequency of lots transportation in order to minimize the total transportation cost. Consequently, the optimal lot sizing $Q^{*}$ increases, and leads to a systematic increase in the optimal hedging level $Z^{*}$ in order to protect the system from backlogs. When the backlog cost decreases (cases 9-10), the optimal lot sizing and the optimal threshold decrease very significantly. It should be noted that, when the transportation costs ( $c_{t}$ and $\left.c_{v}\right)$ are set to zero, the optimal lot sizing is equal to 1 . Such situation leads to item-by-item production system that can be controlled by the classical HPP. Therefore, we can consider that the classical HPP is a particular form of the EMQ-HPP policy when the transportation costs are negligible.

Through the above analysis, it clearly appears that the results make sense, and that the proposed production control policy and resolution approach are robust. In the next section, we study the impact of the transportation delay on the design of the production control policy. 


\subsection{Sensitivity analysis of transportation delay}

Another set of experiments is conducted to measure the sensitivity of the control policy parameters with the respect to transportation delay. Table 3 presents the optimal design factors and the incurred cost variations corresponding to different transportation delay changes.

Table 3. Sensitivity analysis for the transportation delay.

\begin{tabular}{|c|c|c|c|c|c|c|}
\hline \multirow{2}{*}{$\tau$} & \multicolumn{2}{|c|}{ Optimal lot sizing } & \multicolumn{2}{|c|}{ Optimal hedging level } & \multicolumn{2}{|c|}{ Optimal Cost } \\
\hline & $Q^{*}$ & $\Delta Q^{*}(\%)$ & $Z^{*}$ & $\Delta Z^{*}(\%)$ & $C^{*}$ & $\Delta C^{*}(\%)$ \\
\hline $0 \quad(-100 \%)$ & 8912 & $-0.36 \%$ & 20047 & $-56.66 \%$ & 3812.52 & $-23.16 \%$ \\
\hline $1.5(-50 \%)$ & 8923 & $-0.24 \%$ & 25634 & $-22.51 \%$ & 4376.88 & $-11.79 \%$ \\
\hline 3.0 (basic) & 8944 & - & 31405 & - & 4961.68 & - \\
\hline $4.5(+50 \%)$ & 8954 & $+0.11 \%$ & 37307 & $+15.82 \%$ & 5557.50 & $12.01 \%$ \\
\hline $6.0(+100 \%)$ & 8960 & $+0.18 \%$ & 43285 & $+27.45 \%$ & 6157.18 & $24.09 \%$ \\
\hline
\end{tabular}

Although the transportation delay is changed significantly $\left( \pm 100 \%\right.$ ), the optimal lot sizing $Q^{*}$ does not seem to be sensitive to this variation. However, the optimal hedging threshold $Z^{*}$ is considerably sensitive to the change in the transportation delay. In fact, when the transportation delay increases, the lots produced take much time to arrive to the final stock. To ensure more protection against backlogs, the system reacts by increasing the average stock level. The optimal incurred cost is fairly sensitive to the delay change.

\section{Conclusion}

In this paper, we have focused on determining the optimal production control policy and the economic manufacturing quantity for a single-product failure-prone manufacturing systems with transportation delay. In the literature, most of the EMQ models for unreliable manufacturing systems are obtained assuming that the lot under production can instantly meet demand and build a safety stock. This assumption cannot be applied for a wide range of manufacturing systems where lots produced must be transported to a buffer stock before serving demand.

An optimal feedback control policy in which the lot sizing is considered as a decision variable has been developed using a combination of analytical and numerical methods. The policy obtained, named EMQ-HPP, consists of a combination of a modified HPP (to control the production rate) and an inventory-feedback policy (to control production orders). A simulationbased experimental approach is then used to determine the optimal policy parameters $\left(Q^{*}, Z^{*}\right)$. 
Finally, a complete sensitivity analysis is performed in order to confirm that the EMQ-HPP is very closely approximates for the optimal control problem.

In future, this work could be extended in two possible directions in order to control more real and complex industrial problems. The first involves considering the problem of supplying multiple warehouses in different locations, while the second deals with random transportation delays and unreliable transportation networks.

\section{References}

Abboud, N. E. (1997). A simple approximation of the EMQ model with Poisson machine failures. Production Planning \& Control, 8(4), 385-397.

Akella, R., \& Kumar, P. (1986). Optimal control of production rate in a failure prone manufacturing system. IEEE Transactions on Automatic Control, 31(2), 116-126.

Ben-Daya, M. (2002). The economic production lot-sizing problem with imperfect production processes and imperfect maintenance. International Journal of Production Economics, 76(3), 257-264.

Chakraborty, T., Giri, B., \& Chaudhuri, K. (2009). Production lot sizing with process deterioration and machine breakdown under inspection schedule. Omega, 37(2), 257-271.

Chung, K. J. (1997). Bounds for production lot sizing with machine breakdowns. Computers \& Industrial Engineering, 32(1), 139-144.

Ertogral, K., Darwish, M., \& Ben-Daya, M. (2007). Production and shipment lot sizing in a vendor-buyer supply chain with transportation cost. European Journal of Operational Research, 176(3), 1592-1606.

Gershwin, S. B. (1994). Manufacturing systems engineering: Prentice Hall.

Gershwin, S. B. (2000). Design and operation of manufacturing systems: the control-point policy. IIE Transactions, 32(10), 891-906.

Giri, B., \& Dohi, T. (2005). Computational aspects of an extended EMQ model with variable production rate. Computers \& operations research, 32(12), 3143-3161.

Giri, B., \& Yun, W. (2005). Optimal lot sizing for an unreliable production system under partial backlogging and at most two failures in a production cycle. International Journal of Production Economics, 95(2), 229-243. 
Giri, B., Yun, W., \& Dohi, T. (2005). Optimal design of unreliable production-inventory systems with variable production rate. European Journal of Operational Research, 162(2), 372386.

Groenevelt, H., Pintelon, L., \& Seidmann, A. (1992a). Production lot sizing with machine breakdowns. Management Science, 38(1), 104-123.

Groenevelt, H., Pintelon, L., \& Seidmann, A. (1992b). Production batching with machine breakdowns and safety stocks. Operations Research, 40(5), 959-971.

Hajji, A., Gharbi, A., \& Kenné, J. (2009). Joint replenishment and manufacturing activities control in a two stage unreliable supply chain. International Journal of Production Research, 47(12), 3231-3251.

Hariga, M., \& Ben-Daya, M. (1998). Note: the economic manufacturing lot-sizing problem with imperfect production processes: bounds and optimal solutions. Naval research logistics, 45(4), 423-433.

Kelton, W. D., \& Law, A. M. (2000). Simulation modeling and analysis: McGraw Hill.

Kenné, J., Boukas, E., \& Gharbi, A. (2003). Control of production and corrective maintenance rates in a multiple-machine, multiple-product manufacturing system. Mathematical and computer modelling, 38(3-4), 351-365.

Kenné, J., \& Gharbi, A. (1999). Experimental design in production and maintenance control problem of a single machine, single product manufacturing system. International Journal of Production Research, 37(3), 621-637.

Kenné, J., \& Gharbi, A. (2000). Production planning problem in manufacturing systems with general failure and repair time distributions. Production Planning \& Control, 11(6), 581588.

Kim, C. H., \& Hong, Y. (1997). An extended EMQ model for a failure prone machine with general lifetime distribution. International Journal of Production Economics, 49(3), 215223.

Kim, C. H., Hong, Y., \& Kim, S.-Y. (1997). An extended optimal lot sizing model with an unreliable machine. Production Planning \& Control: The Management of Operations, $8(6), 577$ - 585.

Kimemia, J., \& Gershwin, S. B. (1983). An Algorithm for the Computer Control of a Flexible Manufacturing System. IIE Transactions, 15(4), 353 - 362. 
Kushner, H.J. and Dupuis, P.G., 1992. Numerical methods for stochastic control problems in continuous time: Springer-verlag.

Li, J., Sava, A., \& Xie, X. (2009). An analytical approach for performance evaluation and optimization of a two-stage production-distribution system. International journal of production research, 47(2), 403-414.

Liao, G. L., Chen, Y. H., \& Sheu, S. H. (2009). Optimal economic production quantity policy for imperfect process with imperfect repair and maintenance. European Journal of Operational Research, 195(2), 348-357.

Mourani, I., Hennequin, S., \& Xie, X. (2008). Simulation-based optimization of a single-stage failure-prone manufacturing system with transportation delay. International Journal of Production Economics, 112(1), 26-36.

Pegden, C. D., Shannon, R. E., Sadowski, R. P., \& Corp, S. M. (1995). Introduction to simulation using SIMAN: McGraw-Hill.

Salameh, M., \& Jaber, M. (2000). Economic production quantity model for items with imperfect quality. International Journal of Production Economics, 64(1-3), 59-64.

Sana, S. S., \& Chaudhuri, K. (2010). An EMQ model in an imperfect production process. International Journal of Systems Science, 41(6), 635-646.

Sethi, S. P., \& Thompson, G. L. (2005). Optimal control theory: applications to management science and economics: Springer Verlag.

Sethi, S. P., \& Zhang, Q. (1994). Hierarchical decision making in stochastic manufacturing systems: Birkhauser Verlag Basel, Switzerland, Switzerland.

Sharifnia, A. (1988). Production control of a manufacturing system with multiple machine states. IEEE Transactions on Automatic Control, 33(7), 620-625.

Van Ryzin, G. J., Lou, S. X. C., \& Gershwin, S. B. (1991). Scheduling job shops with delays. International Journal of Production Research, 29(7), 1407-1422.

Yang, T. (1999). Impulsive control. Automatic Control, IEEE Transactions on, 44(5), 1081-1083.

Yong, J. (1989). Systems governed by ordinary differential equations with continuous, switching and impulse controls. Applied Mathematics and Optimization, 20(1), 223-235. 